\title{
Spectrum and prevalence of $B R C A 1 / 2$ germline mutations in Pakistani breast cancer patients: results from a large comprehensive study
}

Muhammad Usman Rashid ${ }^{1,2}$, Noor Muhammad ${ }^{1}$, Humaira Naeemi ${ }^{1}$, Faiz Ali Khan ${ }^{1}$, Mariam Hassan³, Saima Faisal ${ }^{3}$, Sidra Gull ${ }^{1,4}$, Asim Amin $^{5}$, Asif Loya ${ }^{6}$ and Ute Hamann ${ }^{2 *}$ (D)

\begin{abstract}
Background: Pathogenic germline mutations in BRCA1 and BRCA2 (BRCA1/2) account for the majority of hereditary breast and/or ovarian cancers worldwide. To refine the spectrum of BRCA1/2 mutations and to accurately estimate the prevalence of mutation in the Pakistani population, we studied 539 breast cancer patients selected for family history and age of diagnosis.

Methods: Comprehensive screening for BRCA1/2 germline mutations was performed using state-of-the-art technologies. Results: A total of 133 deleterious mutations were identified in 539 families (24.7\%), comprising 110 in BRCA1 and 23 in $B R C A 2$. The prevalence of BRCA1/2 small-range mutations and large genomic rearrangements was $55.4 \%$ (36/65) for families with breast and ovarian cancer, 27.4\% (67/244) for families with two or more cases of breast cancer, 18.5\% (5/27) for families with male breast cancer, and 12.3\% (25/203) for families with a single case of early-onset breast cancer. Nine mutations were specific to the Pakistani population. Eighteen mutations in BRCA1 and three in BRCA2 were recurrent and accounted for $68.2 \%$ (75/110) and 34.8\% (8/23) of all identified mutations in BRCA1 and BRCA2, respectively. Most of these mutations were exclusive to a specific ethnic group and may result from founder effects.
\end{abstract}

Conclusions: Our findings show that BRCA1/2 mutations account for one in four cases of hereditary breast/ovarian cancer, one in five cases of male breast cancer, and one in eight cases of early-onset breast cancer in Pakistan. Our study suggests genetic testing of an extended panel of 21 recurrent BRCA1/2 mutations for appropriately selected patients and their families in Pakistan.

Keywords: BRCA1/2, germline mutations, breast cancer, Pakistan

\section{Background}

Individuals harboring $B R C A 1 / 2$ germline mutations have high lifetime risks of breast and ovarian cancer. The identification of individuals harboring BRCA1/2 mutations is crucial to assess their cancer risk, consider preventive measures and tailor cancer management strategies.

Several studies have investigated the prevalence of $B R C A 1 / 2$ small-range mutations and/or large genomic rearrangements (LGRs) with frequencies varying from

\footnotetext{
* Correspondence: u.hamann@dkfz-heidelberg.de

${ }^{2}$ Molecular Genetics of Breast Cancer, German Cancer Research Center

(DKFZ), Im Neuenheimer Feld 580, 69120 Heidelberg, Germany

Full list of author information is available at the end of the article
}

$17.6 \%$ to $29.8 \%$ in white populations from Europe and Australia [1-5] and $9.4 \%$ to $21.7 \%$ in non-whites from Asia [6-8]. The prevalence and distribution of BRCA1/2 mutations vary across populations, mainly due to population-specific recurrent or founder mutations. Accurate identification of the population-specific mutation spectrum is therefore the first step towards incorporating appropriate genetic $B R C A 1 / 2$ testing into clinical practice in a particular population. This information is not fully elucidated in Pakistan, a country with one of the highest rates of breast cancer in Asia.

To date, no large comprehensive studies evaluating the BRCA1/2 mutations have been reported in the Pakistani

(c) The Author(s). 2019 Open Access This article is distributed under the terms of the Creative Commons Attribution 4.0 International License (http://creativecommons.org/licenses/by/4.0/), which permits unrestricted use, distribution, and 
population and mutations in males have not been identified so far. Small-range mutations were previously reported in 341 unselected breast and 120 ovarian cancer patients, in which the analysis was restricted to a few exons only [9]. We conducted two studies in early-onset and familial breast/ovarian cancer patients from Pakistan. In the initial study the complete coding regions and exonintron boundaries of $B R C A 1 / 2$ were screened for smallrange mutations in 176 patients [10]. In the other study 120 BRCA1/2 small-range mutations negative patients were screened for LGRs [11]. Other Asian studies also had small sample sizes [12, 13], reported small-range mutations only $[14,15]$, and/or restricted LGR analyses to a small number of study participants $[6,16,17]$.

Here, we refined the spectrum of $B R C A 1 / 2$ mutations and more precisely estimated the mutation frequencies including small-range mutations and LGRs in 539 earlyonset and familial breast cancer patients from Pakistan.

\section{Methods}

\section{Enrollment of families}

Five hundred and ninety-three breast cancer only or breast and ovarian cancer families were enrolled through index breast and/or ovarian cancer patients who presented at the Shaukat Khanum Memorial Cancer Hospital and Research Centre (SKMCH\&RC) in Lahore, Pakistan, from September 2004 to August 2015. The recruited families were classified into five risk groups based on family history of breast/ovarian cancer or age at diagnosis (Table 1) as described previously [19]. After enrollment, 54 families were excluded (Fig. 1), leaving 539 families in the study.

Clinical and histopathological data and comprehensive information on personal and family history of cancer(s), and ethnicity were obtained from all study participants. The Institutional Review Board of the SKMCH\&RC approved the study. All study participants signed an informed written consent before providing a blood sample.

\section{BRCA1/2 mutation screening}

Genomic DNA was extracted from 9 to $18 \mathrm{ml}$ of whole blood samples, as described previously [20]. The entire coding regions of the $B R C A 1$ (Genbank accession number U14680) and BRCA2 (Genbank accession number U43746) genes including exon-intron boundaries were screened in 139 patient DNA samples for small-range mutations using denaturing high-performance liquid chromatography (DHPLC) analysis as previously described [21, 22]. Each sample revealing variants was sequenced as described elsewhere [18]. Four-hundred female patients selected based on triple-negative vs. non triple-negative breast cancer phenotype had been screened for smallrange $B R C A 1 / 2$ mutations and 33 mutations were described [18]. All small-range mutation-negative patients had been screened for LGRs using multiplex ligationdependent probe amplification and three LGRs were described [11]. For the current study, families were selected on the basis of family history of breast/ovarian cancer, male breast cancer or age at diagnosis.

\section{Mutation classification}

All $B R C A 1 / 2$ alterations identified in the current study were classified into pathogenic mutations, variants of unknown significance, or polymorphisms. Pathogenic mutations were defined as (i) small-range mutations which affect one or a few nucleotides including frameshift, nonsense, or splice-site mutations and generate a premature termination codon, except BRCA2 exon 27 variants generating a premature termination codon after codon 3010 [23] and (ii) LGRs that span one or more exons. Mutations were designated using the Human

Table 1 BRCA1/2 mutation frequencies according to family structure

\begin{tabular}{|c|c|c|c|c|c|c|c|c|c|}
\hline \multirow{3}{*}{$\begin{array}{l}\text { Risk } \\
\text { group }\end{array}$} & \multirow[t]{3}{*}{ Phenotype of families } & \multirow{3}{*}{$\begin{array}{l}\text { No. of } \\
\text { families }\end{array}$} & \multicolumn{7}{|c|}{ No. of families with mutations (\%) in } \\
\hline & & & \multicolumn{3}{|c|}{$B R C A 1$} & \multicolumn{3}{|c|}{ BRCA2 } & \multirow[t]{2}{*}{$B R C A 1 / 2$} \\
\hline & & & Small-range & LGRs & All & Small-range & LGRS & All & \\
\hline & All families & 539 & $101(18.7)$ & $9(1.7)$ & $110(20.4)$ & $23(4.3)$ & $0(0)$ & $23(4.3)$ & $133(24.7)^{a}$ \\
\hline & Female breast cancer families & 447 & $67(15.0)$ & $7(1.6)$ & $74(16.6)$ & $18(4.0)$ & $0(0)$ & $18(4.0)$ & $92(20.6)$ \\
\hline $\mathrm{A} 1$ & 1 case $\leq 30$ years & 203 & $20(9.8)$ & $2(1.0)$ & $22(10.8)$ & $3(1.5)$ & $0(0)$ & $3(1.5)$ & $25(12.3)$ \\
\hline$A 2$ & 2 cases, $\geq 1$ diagnosed $\leq 50$ years & 131 & $20(15.3)$ & $4(3.0)$ & $24(18.3)$ & $6(4.6)$ & $0(0)$ & $6(4.6)$ & $30(22.9)$ \\
\hline A3 & $\geq 3$ cases, $\geq 1$ diagnosed $\leq 50$ years & 113 & $27(23.9)$ & $1(0.9)$ & $28(24.8)$ & $9(8.0)$ & $0(0)$ & $9(8.0)$ & $37(32.7)$ \\
\hline \multirow[t]{2}{*}{ A4 } & Male breast cancer families & & & & & & & & \\
\hline & $\geq 1$ case of male breast cancer & 27 & $1(3.7)$ & $0(0)$ & $1(3.7)$ & $4(14.8)$ & $0(0)$ & $4(14.8)$ & $5(18.5)$ \\
\hline \multirow[t]{2}{*}{ B } & Breast-ovarian cancer families & & & & & & & & \\
\hline & $\geq 1$ breast cancer and $\geq 1$ ovarian cancer & 65 & $33(50.8)$ & $2(3.0)$ & $35(53.8)$ & $1(1.5)$ & $0(0)$ & $1(1.5)$ & $36(55.4)$ \\
\hline
\end{tabular}




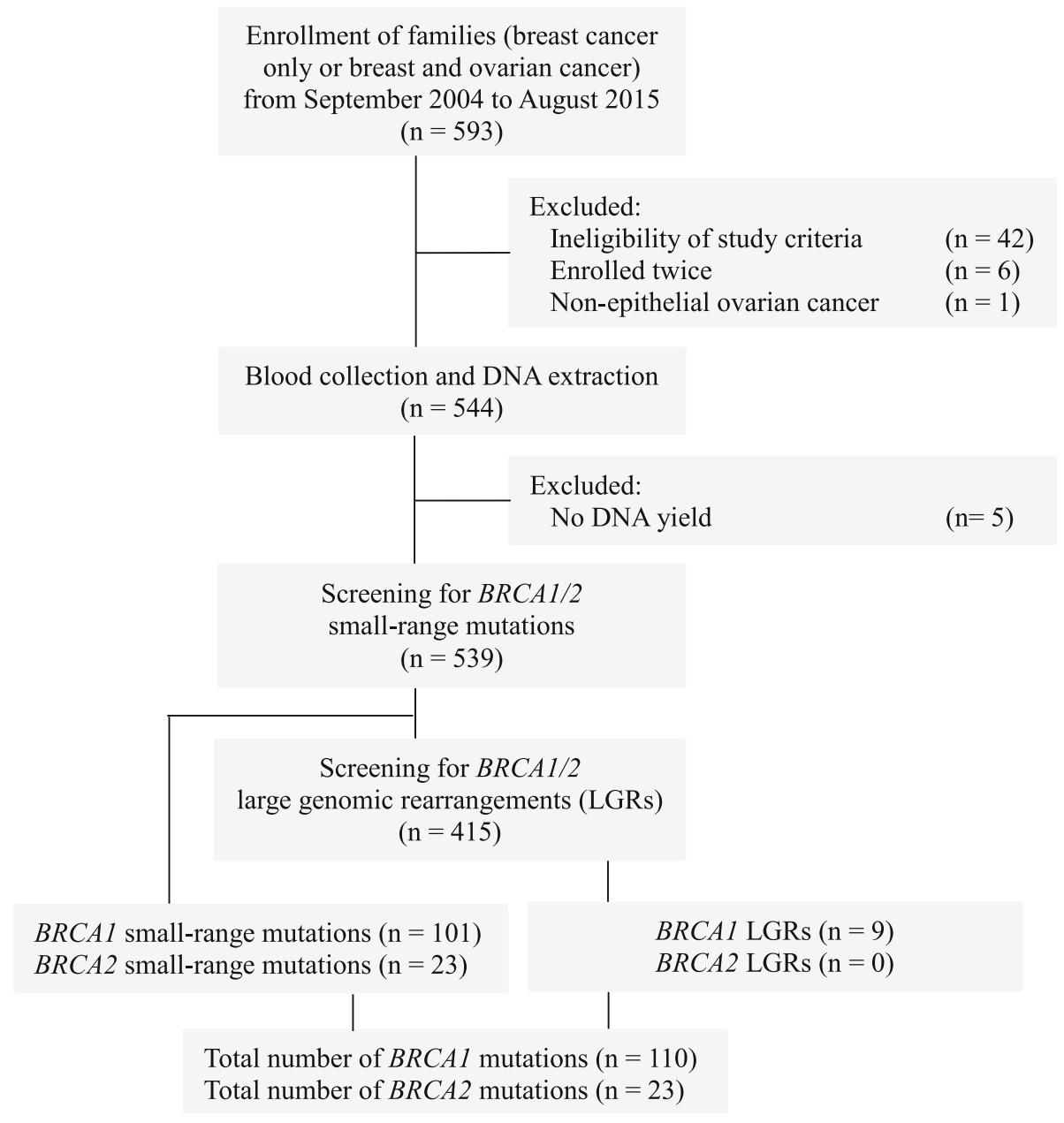

Fig. 1 Flow diagram for the study participant's enrollment, screening methods used and BRCA1/2 mutations detected

Genome Variation Society (HGVS) and the Breast Cancer Information Core (BIC) committee nomenclature.

All identified mutations were searched in various mutation databases including BIC (https://research.nhgri.nih. gov/bic/), ClinVar (http://www.ncbi.nim.nih.gov/clinvar/), LOVD (http://databases.lovd.nl/shared/genes/BRCA2), ARUP (http://arup.utah.edu/database/BRCA/), and BRCA Exchange (http://brcaexchange.org/). Mutations not reported in these databases were considered as novel and specific to Pakistani population.

\section{Statistical analyses}

Distribution of clinical and histopathological characteristics between BRCA1/2 carriers and non-carriers were estimated using Fisher's exact test for categorical variables and the Wilcoxon rank-sum test for quantitative variables. All statistical tests were two-sided. Results were considered significant at a $p$ value of $\leq 0.05$. All statistical analyses were done using StatXact 4 for Windows (Cytel, Cambridge, USA) and R, version 2.1.

\section{Results}

A total of 539 index patients from unrelated families were enrolled and stratified into five risk groups (Table 1). The mean age of disease onset was 35.4 years (range 18-78) for female breast cancer $(n=502), 45.4$ years (range 23-66) for ovarian cancer $(n=30)$ and 54.5 years (range 27-76) for male breast cancer $(n=27)$ patients.

\section{Spectrum of $B R C A 1 / 2$ mutations}

Evaluation of pooled data from 539 patients yielded 71 distinct pathogenic mutations in 133 families (24.7\%) (Table 1). Fifty-three BRCA1 mutations were detected in 110 families (20.4\%) and 18 BRCA2 mutations in 23 families (4.3\%). Five mutations in BRCA1 (9.4\%) and four mutations in BRCA2 (22.2\%) were novel (Table 2). The phenotypes of all families carrying BRCA1/2 mutations are presented in Table 3.

Twenty-one $(21 / 71 ; 29.6 \%)$ mutations including 18 in BRCA1 and three in BRCA2 occurred more than once (Fig. 2a, b). These mutations were identified in 83 
Table 2 Deleterious BRCA1/2 germline mutations in Pakistani breast/ovarian cancer families

\begin{tabular}{|c|c|c|c|c|c|c|c|c|}
\hline \multirow[t]{2}{*}{ Family } & \multirow[t]{2}{*}{ Exon } & \multicolumn{3}{|c|}{ BIC designation } & \multicolumn{2}{|c|}{ HGVS designation } & \multirow{2}{*}{$\begin{array}{l}\text { Mutation } \\
\text { type }^{b}\end{array}$} & \multirow{2}{*}{$\begin{array}{l}\text { Reported } \\
\text { in } \\
\text { databases } \\
\text { (No. of }_{\text {entries) }}^{c}\end{array}$} \\
\hline & & Nucleotide & Codon & Designation & $\begin{array}{l}\text { Nucleotide } \\
\text { change }^{a}\end{array}$ & Effect on protein & & \\
\hline \multicolumn{9}{|l|}{ BRCA1-small-range mutations } \\
\hline 432 & 2 & 185 & 23 & 185insA & c.66dup & p.(Glu23Argfs*18) & FS & $B I C(32)^{d}$ \\
\hline 723 & 2 & 185 & 23 & 185delAG & c.68_69del & p.(Glu23Valfs*17) & FS & $\begin{array}{l}\text { BIC } \\
(2036)^{d}\end{array}$ \\
\hline 372 & $\begin{array}{l}\text { Intron } \\
4\end{array}$ & IVS4-2 & - & IVS4-2A>G & c. $135-2 A>G$ & Splice site & SP & $\mathrm{BIC}(1)$ \\
\hline $254^{e}$ & 7 & 454 & 112 & 454delA & c.335del & p.(Asn112llefs*7) & FS & ClinVar (2) \\
\hline 449 & 7 & 509 & 130 & Y130X & c. $390 C>G$ & p.(Tyr130*) & NS & LOVD (3) \\
\hline $\begin{array}{l}296,317,340^{\mathrm{e}}, 511,521,626^{\mathrm{e}} \\
747\end{array}$ & 11 & 804 & 229 & 804delT & c.685del & p.(Ser229Leuf**5) & FS & $\mathrm{BIC}(2)$ \\
\hline $470^{e}$ & 11 & 903 & 262 & Q262X & c.784C $>\mathrm{T}$ & p. $\left(G \ln 262^{*}\right)$ & NS & ClinVar (3) \\
\hline 711 & 11 & 1014 & 299 & 1014delGT & c.895_896del & p.(Val299Argfs*4) & FS & $\mathrm{BIC}(2)$ \\
\hline $669^{e}$ & 11 & 1127 & 336 & 1127delA & c.1008del & p.(Glu337Lysfs*4) & FS & $\mathrm{No}^{d}$ \\
\hline 748 & 11 & 1307 & 396 & 1307delT & c.1188del & p.(Asp396Glufs*14) & FS & LOVD (1) \\
\hline $241^{e}$ & 11 & 1309 & 397 & 1309delA & c.1190del & p.(Asp397Alafs*13) & FS & ClinVar (3) \\
\hline 722 & 11 & 1518 & 485 & 1518_1572dup55 & c.1399_1453dup & p.(Ala485Glufs*13) & FS & No \\
\hline $336^{e}$ & 11 & 1590 & 491 & Q491X & c. $1471 C>T$ & p.(Gln491*) & NS & $B I C(4)^{d}$ \\
\hline $\mathrm{N} 12$ & 11 & 1898 & 593 & 1898delTATGGAA & c.1779_1785del & p.(Met594Serfs*3) & FS & LOVD (2) \\
\hline $\mathrm{N} 28,328^{\mathrm{e}}, 557^{\mathrm{e}}$ & 11 & 1912 & 598 & L598X & c. $1793 \mathrm{~T}>\mathrm{G}$ & p.(Leu598*) & NS & $\operatorname{BIC}(1)^{d}$ \\
\hline $574^{e}$ & 11 & 2080 & 655 & 2080ins A & c.1961dup & p.(Tyr655Valfs*18) & FS & BIC $(13)^{d}$ \\
\hline $488^{e}$ & 11 & 2268 & 717 & E717X & $c .2149 G>T$ & p. $($ Glu717*) & NS & ClinVar (2) \\
\hline $236^{\mathrm{e}}, 283^{\mathrm{e}}, 489^{\mathrm{e}}, 493^{\mathrm{e}}, 593$ & 11 & 2388 & 757 & 2388delG & c.2269del & p.(Val757Phefs*8) & FS & $B I C(10)^{d}$ \\
\hline 363 & 11 & 2433 & 772 & 2433delGT & c.2314_2315del & p.(Val772Thrfs*4) & FS & LOVD (2) \\
\hline $550^{h}$ & 11 & 2457 & 780 & Q780X & $c .2338 C>T$ & p. $\left(G \ln 780^{*}\right)$ & NS & $\mathrm{BIC}(36)$ \\
\hline 362,469 & 11 & 2459 & 780 & 2459delGGAA & c.2340_2343del & p.(Glu781Valfs*10) & FS & LOVD (2) \\
\hline $421^{\mathrm{e}}, 442,510^{\mathrm{e}}, 542,619^{\mathrm{e}}$ & 11 & 2524 & 802 & 2524delTG & c.2405_2406del & p.(Val802Glufs*7) & FS & $B I C(5)^{d}$ \\
\hline N34 & 11 & 2657 & 846 & 2657delAAT-insG & $\begin{array}{l}\text { c.2538_- } \\
\text { 2540delinsG }\end{array}$ & p.(Met847Glyfs*4) & FS & LOVD (2) \\
\hline $415^{\mathrm{e}}, 660^{\mathrm{e}}$ & 11 & 2722 & 868 & S868X & c. $2603 C>G$ & p.(Ser868*) & NS & $\operatorname{BIC}(11)^{d}$ \\
\hline $411^{\mathrm{e}}$ & 11 & 3090 & 991 & K991X & c. $2971 \mathrm{~A}>\mathrm{T}$ & p.(Lys991*) & NS & ClinVar (2) \\
\hline $247^{e}$ & 11 & 3100 & 994 & 3100delGT & c.2981_2982del & p.(Cys994*) & FS & ClinVar (5) \\
\hline 299 & 11 & 3248 & 1043 & 3248delTATTAATGAA & c.3129_3138del & p.(Asn 1043Lysfs*2) & FS & ClinVar (3) \\
\hline $\mathrm{N} 13^{\mathrm{e}}, 399^{\mathrm{e}}$ & 11 & 3458 & 1113 & 3458delTGA & c.3339_3341del & p.(Tyr1113*) & FS & ClinVar (2) \\
\hline 610 & 11 & 3531 & 1138 & G1138X & $c .3412 \mathrm{G}>\mathrm{T}$ & p.(Gly1138*) & NS & ClinVar (3) \\
\hline N25 & 11 & 3596 & 1159 & 3596delAAAG & c.3477_3480del & p.(Ile1159Metfs*50) & FS & $\mathrm{BIC}(3)$ \\
\hline $279^{e}, 445^{e}$ & 11 & 3717 & 1200 & Q1200X & c.3598C >T & p. $\left(G \ln 1200^{*}\right)$ & NS & $\operatorname{BIC}(21)^{d}$ \\
\hline 382 & 11 & 3726 & 1203 & R1203X & c.3607C $>T$ & p. $\left(\operatorname{Arg} 1203^{*}\right)$ & NS & $\mathrm{BIC}(36)$ \\
\hline 646 & 11 & 3819 & 1234 & 3819delGTAAA & c.3700_3704del & p.(Val1234Glnfs*8) & FS & $\mathrm{BIC}(61)$ \\
\hline $\begin{array}{l}210^{\mathrm{e}}, 211^{\mathrm{e}}, 313^{\mathrm{e}}, 316,332^{\mathrm{e}}, 463 \\
520,624,652^{\mathrm{e}}, 653\end{array}$ & 11 & 3889 & 1257 & 3889delAG & c.3770_3771del & p.(Glu1257Glyfs*9) & FS & BIC $(23)^{d}$ \\
\hline$N 4^{e}, 687,724,743$ & 11 & 4184 & 1355 & 4184delTCAA & c.4065_4068del & p.(Asn1355Lysfs*10) & FS & BIC $(144)^{d}$ \\
\hline $318^{e}$ & 12 & 4302 & 1395 & Q1395X & c. $4183 C>T$ & p. $\left(G \ln 1395^{*}\right)$ & NS & $\operatorname{BIC}(28)^{d}$ \\
\hline $408^{e}$ & 13 & 4446 & 1443 & R1443X & $c .4327 C>T$ & p. $\left(\operatorname{Arg} 1443^{*}\right)$ & NS & $\mathrm{BIC}(128)$ \\
\hline
\end{tabular}


Table 2 Deleterious BRCA1/2 germline mutations in Pakistani breast/ovarian cancer families (Continued)

\begin{tabular}{|c|c|c|c|c|c|c|c|c|}
\hline \multirow[t]{2}{*}{ Family } & \multirow[t]{2}{*}{ Exon } & \multicolumn{3}{|c|}{$\mathrm{BIC}$ designation } & \multicolumn{2}{|c|}{ HGVS designation } & \multirow{2}{*}{$\begin{array}{l}\text { Mutation } \\
\text { type }^{b}\end{array}$} & \multirow{2}{*}{$\begin{array}{l}\text { Reported } \\
\text { in } \\
\text { databases } \\
\text { (No. of }_{\text {entries) }}^{c}\end{array}$} \\
\hline & & Nucleotide & Codon & Designation & $\begin{array}{l}\text { Nucleotide } \\
\text { change }^{\mathrm{a}}\end{array}$ & Effect on protein & & \\
\hline $523^{\mathrm{e}}, 555, \mathrm{~N} 18,598^{\mathrm{e}}, 612,621$ & $\begin{array}{l}\text { Intron } \\
14\end{array}$ & IVS14-1 & - & IVS14-1G>A & c. $4485-1 G>A$ & Splice site & $\mathrm{SP}$ & $B I C(2)^{d}$ \\
\hline $220^{\mathrm{e}}, 275^{\mathrm{e}}, 512^{\mathrm{e}}$ & 15 & 4627 & 1503 & S1503X & C. $4508 C>A$ & p.(Ser1503*) & NS & $B \mid C(1)^{d}$ \\
\hline $609^{e}$ & 15 & 4784 & 1558 & 4784delG & c.4665del & p.(Arg1555Serfs*4) & FS & No \\
\hline $611^{\mathrm{e}}$ & 16 & 4981 & 1621 & 4981delA & c.4862del & p.(Asp1621Valfs*12) & FS & No \\
\hline $249^{e}, 658$ & 17 & 5154 & 1679 & 5154delC & c.5035del & p.(Leu1679*) & FS & $\mathrm{BIC}(2)$ \\
\hline $276^{\mathrm{e}}, 679$ & $\begin{array}{l}\text { Intron } \\
17\end{array}$ & IVS17+1 & - & IVS17+1G>A & c. $5074+1 \mathrm{G}>\mathrm{A}$ & Splice site & $\mathrm{SP}$ & $\mathrm{BIC}(3)$ \\
\hline 685 & 20 & 5358 & 1747 & 5358delC & c.5239del & p.(Gln1747Lysfs*18) & FS & LOVD (2) \\
\hline 734 & 20 & 5385 & 1756 & 5385dupC & c.5266dup & p.(Gln1756Profs*74) & FS & $\begin{array}{l}\text { LOVD } \\
(376)\end{array}$ \\
\hline 706 & $\begin{array}{l}\text { Intron } \\
20\end{array}$ & IVS20-1 & - & IVS20-1G>C & c.5278-1G>C & Splice site & $\mathrm{SP}$ & $\operatorname{LOVD}(5)^{\mathrm{d}}$ \\
\hline 678 & 21 & 5429 & 1771 & 5429dupG & c.5310dup & p.(Pro1771Alafs*59) & FS & LOVD (1) \\
\hline $278,338^{e}$ & 22 & 5480 & 1787 & 5480delTG & c.5361_5362del & p.(Cys1787Trpfs*42) & FS & ClinVar (3) \\
\hline 682 & 22 & 5496 & 1793 & K1793X & C.5377A $>T$ & p.(Lys1793*) & NS & ClinVar (1) \\
\hline $248^{e}$ & $\begin{array}{l}\text { Intron } \\
23\end{array}$ & IVS23-2 & - & IVS23-2A>T & c. $5468-2 \mathrm{~A}>\mathrm{T}$ & Splice site & SP & ClinVar (1) \\
\hline $\begin{array}{l}260,264,329^{\mathrm{e}}, 377^{\mathrm{e}}, 389,439 \\
481,501,522\end{array}$ & 24 & 5622 & 1835 & R1835X & C. $5503 C>T$ & p. $\left(\operatorname{Arg} 1835^{*}\right)$ & NS & BIC $(74)^{d}$ \\
\hline \multicolumn{9}{|c|}{ BRCA1-large genomic rearrangements ${ }^{\mathrm{e}}$} \\
\hline $\begin{array}{l}229,291,314,379,406,498 \\
549\end{array}$ & $1-2$ & - & - & del exon 1-2 & $\begin{array}{l}\text { g.41271967 } \\
\text { 41308900 delf }\end{array}$ & & LGR & $(42)^{9}$ \\
\hline 261,719 & $21-24$ & - & - & del exon 21-24 & $\begin{array}{l}\text { g.41172653 } \\
\text { 41205744del }\end{array}$ & & LGR & No \\
\hline \multicolumn{9}{|l|}{ BRCA2-small-range mutations } \\
\hline 497,700 & 3 & 320 & 31 & W31X & $c .92 \mathrm{G}>\mathrm{A}$ & p.(Trp31*) & NS & ClinVar (4) \\
\hline N26 & $\begin{array}{l}\text { Intron } \\
4\end{array}$ & IVS4-2 & - & IVS4-2A>G & c. $426-2 A>G$ & Splice site & $\mathrm{SP}$ & ClinVar (4) \\
\hline 545 & 9 & 993 & 255 & 993delCACAA & c.765_769del & p.(Asn255Lysfs*19) & FS & No \\
\hline 330 & 10 & 1528 & 434 & 1528deIAAAA & c.1300_1303del & p.(Lys434Glufs*25) & FS & ClinVar (2) \\
\hline 602 & 11 & 3048 & 941 & 3048delA & c.2820del & p.(Val941Cysfs*19) & FS & No \\
\hline 206 & 11 & 3063 & 945 & 3063delA & c.2835del & p.(Asp946llefs*14) & FS & ClinVar (2) \\
\hline 505 & 11 & 4088 & 1287 & 4088delA & c.3860del & p.(Asn1287llefs*6) & FS & $\mathrm{BIC}(2)$ \\
\hline $222,407^{h}, 525,540^{h}$ & 11 & 5450 & 1741 & 5450delGTAA & c.5222_5225del & p.(Ser1741Thrfs*35) & FS & $\mathrm{BIC}(1)$ \\
\hline 627,684 & 11 & 5910 & 1894 & Y1894X & c. $5682 C>A$ & p.(Tyr1894*) & NS & $\mathrm{BIC}(3)$ \\
\hline $295^{\mathrm{e}}$ & 11 & 5950 & 1908 & 5950delCT & c.5722_5723del & p.(Leu1908Argfs*2) & FS & BIC $(43)^{d}$ \\
\hline 447 & 11 & 6696 & 2156 & 6696delTC & c.6468_6469del & p.(Gln2157llefs*18) & FS & BIC $(24)^{d}$ \\
\hline $548^{h}$ & 11 & 7044 & 2274 & 7044delAAGAG & c.6816_6820del & p.(Gly2274Alafs*17) & FS & ClinVar (6) \\
\hline 579 & 15 & 7803 & 2526 & 7803 delA & c.7575del & p.(Ala2526Glnfs*2) & FS & LOVD (2) \\
\hline 492 & $\begin{array}{l}\text { Intron } \\
17\end{array}$ & IVS17+2 & - & IVS17+2C>A & c. $7976+2 C>A$ & Splice site & SP & ClinVar (1) \\
\hline 713 & 20 & 8773 & 2849 & 8773delAA & c.8545_8546del & p.(Lys2849Glyfs*19) & FS & No \\
\hline 702 & 20 & 8779 & 2860 & 8779_8798dup20 & c.8551_8570dup & p.(Lys2860Asnfs*10) & FS & No \\
\hline $207^{h}$ & 21 & 8897 & 2890 & 8897insT & c.8669dup & p.(Thr2891Asnfs*16) & FS & ClinVar (1) \\
\hline
\end{tabular}


Table 2 Deleterious BRCA1/2 germline mutations in Pakistani breast/ovarian cancer families (Continued)

\begin{tabular}{|c|c|c|c|c|c|c|c|c|}
\hline \multirow[t]{2}{*}{ Family } & \multirow[t]{2}{*}{ Exon } & \multicolumn{3}{|c|}{$\mathrm{BIC}$ designation } & \multicolumn{2}{|c|}{ HGVS designation } & \multirow{2}{*}{$\begin{array}{l}\text { Mutation } \\
\text { type }^{b}\end{array}$} & \multirow{2}{*}{$\begin{array}{l}\text { Reported } \\
\text { in } \\
\text { databases } \\
\text { (No. of }_{\text {entries) }}^{c}\end{array}$} \\
\hline & & Nucleotide & Codon & Designation & $\begin{array}{l}\text { Nucleotide } \\
\text { change }^{a}\end{array}$ & Effect on protein & & \\
\hline 538 & $\begin{array}{l}\text { Intron } \\
21\end{array}$ & IVS21+4 & - & IVS21+4A>G & c. $8754+4 A>G$ & Splice site & SP & $\mathrm{BIC}(7)$ \\
\hline
\end{tabular}

${ }^{a}$ Numbering starts at the first A of the first coding ATG (located in exon 2) of NCBI GenBank accession number U14680 (BRCA1) and U43746 (BRCA2)

${ }^{\mathrm{b}}$ FS frameshift mutation, LGR large genomic rearrangement, MS missense mutation, NS nonsense mutation, SP splice-site mutation

${ }^{\mathrm{C}}$ BIC Breast Cancer Information Core database (https://research.nhgri.nih.gov/projects/bic/), LOVD Leiden Open Variation Database (http://databases.lovd.nl/shared/ genes/BRCA2); ClinVar (https://www.ncbi.nlm.nih.gov/clinvar/), date last accessed June 26, 2018

dPreviously reported in Pakistani breast/ovarian cancer cases $[9,10]$

e Families and mutation description have been previously reported $[11,18]$

f Genomic locale for chromosome 17, from the UCSC genome browser, Feb 2009 assembly

${ }^{9}$ Not available in databases; reported in various studies $[1,11]$

${ }^{\mathrm{h}}$ Families with male breast cancer

unrelated families and accounted for $62.4 \%$ (83/133) of all families with mutations. The most common BRCA1 mutation was c.3770_3771del (ten Punjabi families), followed by c.5503C $>\mathrm{T}$ (nine Punjabi families), exon 1-2 deletion (seven Punjabi families), c.685del (five Punjabi and two Balochi families), c.4485-1G>A (six Punjabi families), c.2269del (one Punjabi and four Pathan families), c.2405 2406del (five Punjabi families), c.4065_4068del (three Punjabi and one Pathan families), c.1793T $>$ G and c.4508C $>$ A (three Punjabi families each), exon 21-24 deletion, c.2603C > G, c.3339_3341del, c.3598C > T, c.5035del, c.5074+1G>A, and c.5361_5362del (two Punjabi families each) and c.2340_2343del (one Pathan and one Mohajir families). The most common BRCA2 mutation was c.5222_5225del (one Punjabi and three Mohajir families), followed by c.92G $>$ A (two Punjabi families) and c.5682C $>$ A (two Pathan families).

In addition to the deleterious mutations, $153(28.4 \%)$ distinct BRCA1/2 sequence variants were detected: 79 missense variants, 48 non-coding variants, 24 synonymous variants, one in-frame deletion, and one polymorphic nonsense variant in exon 27 of BRCA2 (data not shown).

\section{$B R C A 1 / 2$ mutation frequencies}

The frequencies of $B R C A 1 / 2$ mutations by risk group are provided in Table 1 . For $B R C A 1$, the highest mutation frequency was noted in families with breast and ovarian cancer (53.8\%), followed by families with at least three breast cancer cases (24.8\%), families with two breast cancer cases $(18.3 \%)$, or families with one earlyonset breast cancer case ( $\leq 30$ years) $(10.8 \%)$. For BRCA2, the highest frequency was observed in families with male breast cancer (14.8\%).

\section{Patient and tumors characteristics by BRCA1/2 status}

$B R C A 1$ carriers $(\mathrm{n}=110)$ were more often identified among female patients $(99.1 \%$ vs. $94.6 \%, p=0.039)$ and belonged to the Punjabi ethnic group $(81.8 \%$ vs. $68.7 \%, p=$ 0.030) compared to non-carriers $(n=406)$. In contrast,
BRCA2 carriers $(\mathrm{n}=23)$ were more common among male patients $(17.4 \%$ vs. $5.4 \%, p=0.043)$ and more often belonged to Pathan ethnic group (34.8\% vs. $15.5 \%, p=$ 0.009).

Female breast cancer patients with mutations in BRCA1 $(\mathrm{n}=106)$ or BRCA2 $(\mathrm{n}=19)$ had a similar mean age of diagnosis (34.0 years (range 21-72) and 37.7 years (range 23-56), respectively, $p=0.073$, Wilcoxon rank-sum test), which did not differ to that of non-carriers $(\mathrm{n}=377)$ (35.7 years (range 18-78). In contrast, male breast cancer patients harboring BRCA2 mutations $(\mathrm{n}=4)$ had an older mean age of diagnosis than non-carriers $(n=22)(66.5$ years (range 54-76) and 52.5 years (range 27-69) years, respectively, $p=0.039$, Wilcoxon rank-sum test).

$B R C A 1$-associated breast tumors more often were invasive ductal carcinomas $(99.0 \%$ vs. $91.4 \%, p=0.004)$, triple-negative ( $60.8 \% v s .22 .6 \%, p=<0.0001)$, and of higher tumor grade (grade 3: $94.9 \%$ vs. $63.2 \%, p=<0.0001$ ) compared to tumors of non-carriers. BRCA2-associated breast tumors more often were PR positive compared to tumors of non-carriers $(81.8 \%$ vs. $57.2 \%, p=0.025)$ (data not shown).

\section{Discussion}

To our knowledge, this is the largest Pakistani study that comprehensively investigated the spectrum of $B R C A 1 / 2$ small-range mutations and LGRs and prevalence of mutations in 539 high-risk families. Mutations were identified in 24.7\% (133/539) of families. Eighteen BRCA1 and three $B R C A 2$ mutations were recurrent and accounted for $68.2 \%$ and $34.8 \%$ of all mutations in BRCA1 and BRCA2, respectively. Nine mutations were specific to the Pakistani population, whereas other mutations had been reported elsewhere.

The most common type of identified mutations were frameshift mutations (60.6\%) followed by nonsense mutations (25.4\%). These data are consistent with our previous report [10] and a recent worldwide study [25]. In Pakistani patients, BRCA1 mutations were about 5- 
Table 3 Characteristics of the 133 families with deleterious BRCA1/2 mutations

\begin{tabular}{|c|c|c|c|c|c|c|}
\hline \multirow[t]{2}{*}{ Family } & \multicolumn{2}{|c|}{ No. of cancers } & \multicolumn{2}{|c|}{ Age at onset (years) } & \multirow{2}{*}{$\begin{array}{l}\text { Other cancer(s) } \\
\text { (age at onset in years) }\end{array}$} & \multirow[t]{2}{*}{ Ethnicity } \\
\hline & Female BC (Bilateral) & $\mathrm{OC}(\mathrm{OC}+\mathrm{BC})$ & $\mathrm{BC}$ & OC & & \\
\hline \multicolumn{7}{|c|}{ Families carrying $B R C A 1$-small-range mutations } \\
\hline $236^{a}$ & 1 & - & $22^{\mathrm{b}}$ & - & - & Pathan \\
\hline 316 & 1 & - & $25^{\mathrm{b}}$ & - & - & Punjabi \\
\hline 264 & 1 & - & $26^{\mathrm{b}}$ & - & - & Punjabi \\
\hline 706 & 1 & - & $26^{\mathrm{b}}$ & - & Uterus (67) & Punjabi \\
\hline $\mathrm{N} 12$ & 1 & - & $26^{\mathrm{b}}$ & - & - & Punjabi \\
\hline 624 & 1 & - & $27^{\mathrm{b}}$ & - & - & Punjabi \\
\hline N25 & 1 & - & $28^{\mathrm{b}}$ & - & - & Punjabi \\
\hline $276^{a}$ & 1 & - & $28^{\mathrm{b}}$ & - & - & Punjabi \\
\hline 610 & 1 & - & $28^{\mathrm{b}}$ & - & - & Punjabi \\
\hline 678 & 1 & - & $28^{\mathrm{b}}$ & - & - & Punjabi \\
\hline $411^{\mathrm{a}}$ & 1 & - & $29^{b}$ & - & Stomach (70) & Punjabi \\
\hline 724 & 1 & - & $29^{b}$ & - & $\begin{array}{l}\text { Renal (48), lung (65), } \\
\text { throat (65), unknown }\end{array}$ & Punjabi \\
\hline N28 & 1 & - & $30^{b}$ & - & - & Punjabi \\
\hline $279^{a}$ & $1(1)$ & - & $27 / 36^{b}$ & - & - & Punjabi \\
\hline 278 & 2 & - & $25^{\mathrm{b}}, 32$ & - & - & Kashmiri \\
\hline $332^{\mathrm{a}}$ & 2 & - & $26^{\mathrm{b}}, 51$ & - & Leukemia (45) & Punjabi \\
\hline 682 & 2 & - & $28^{\mathrm{b}}, 40$ & - & $\begin{array}{l}\text { Uterus }(<62,65) \text {, } \\
\text { throat }(<72)\end{array}$ & Punjabi \\
\hline N18 & 2 & - & $29^{b},<50$ & - & - & Punjabi \\
\hline $421^{a}$ & 2 & - & $30^{\mathrm{b}}, 33$ & - & - & Punjabi \\
\hline 432 & 2 & - & $30^{\mathrm{b}}, 53$ & - & Skin (12), oral (54) & Punjabi \\
\hline 520 & 2 & - & $30^{\mathrm{b}}, 47$ & - & Uterus (32) & Punjabi \\
\hline 449 & 2 & - & $32^{\mathrm{b}}, 55$ & - & - & Punjabi \\
\hline $557^{\mathrm{a}}$ & 2 & - & $32^{\mathrm{b}}, 45$ & - & $\begin{array}{l}\text { Unknown }(<55) \text {, } \\
\text { renal }(70)\end{array}$ & Punjabi \\
\hline 747 & 2 & - & $33^{b}, 38$ & - & - & Unknown \\
\hline 722 & 2 & - & $20,34^{b}$ & - & Unknown $(<18, \leq 40)$ & Punjabi \\
\hline 687 & 2 & - & $37^{b}, 45$ & - & - & Punjabi \\
\hline $470^{a}$ & 2 & - & $40^{\mathrm{b}}, 40$ & - & $\begin{array}{l}\text { Stomach (46), colon } \\
\text { (59), lung }\end{array}$ & Punjabi \\
\hline $510^{\mathrm{a}}$ & 2 & - & $40^{b}, 55$ & - & - & Punjabi \\
\hline $\mathrm{N} 13^{\mathrm{a}}$ & 2 & - & $40^{b},>50$ & - & - & Punjabi \\
\hline 593 & 2 & - & $43,44^{b}$ & - & Leukemia (22) & Pathan \\
\hline 299 & $2(1)$ & - & $24 / 27^{b}, 55$ & - & - & Punjabi \\
\hline $660^{a}$ & $2(1)$ & - & $25 / 26^{b}, 70$ & - & Bladder & Punjabi \\
\hline 260 & $2(1)$ & - & $25 / 26^{b}, 28$ & - & - & Punjabi \\
\hline 511 & $2(1)$ & - & $30 / 33^{b},<32$ & - & Brain (75) & Punjabi \\
\hline N34 & 3 & - & $24^{b},<30,31$ & - & - & Punjabi \\
\hline $669^{a}$ & 3 & - & $25^{\mathrm{b}},<40,<50$ & - & Brain $(<78)$, oral $(<80)$ & Punjabi \\
\hline 685 & 3 & - & $26^{\mathrm{b}}, 26, ?$ & - & Blood (2x) & Mohajir \\
\hline 723 & 3 & - & $28^{\mathrm{b}}, 40, ?$ & - & - & Pathan \\
\hline 612 & 3 & - & $29^{b},<30,40$ & - & Throat (45), uterus (48) & Punjabi \\
\hline $313^{\mathrm{a}}$ & 3 & - & $30^{b}, 48, ?$ & - & - & Punjabi \\
\hline
\end{tabular}


Table 3 Characteristics of the 133 families with deleterious BRCA1/2 mutations (Continued)

\begin{tabular}{|c|c|c|c|c|c|c|}
\hline \multirow[t]{2}{*}{ Family } & \multicolumn{2}{|c|}{ No. of cancers } & \multicolumn{2}{|c|}{ Age at onset (years) } & \multirow{2}{*}{$\begin{array}{l}\text { Other cancer(s) } \\
\text { (age at onset in years) }\end{array}$} & \multirow[t]{2}{*}{ Ethnicity } \\
\hline & Female BC (Bilateral) & $\mathrm{OC}(\mathrm{OC}+\mathrm{BC})$ & $\mathrm{BC}$ & OC & & \\
\hline $336^{a}$ & 3 & - & $23,30^{\mathrm{b}}, 38$ & - & Prostate (29) & Punjabi \\
\hline $493^{\mathrm{a}}$ & 3 & - & $35^{\mathrm{b}}, 55,>55$ & - & - & Pathan \\
\hline 382 & 3 & - & $36^{\mathrm{b}}, 50, ?$ & - & - & Punjabi \\
\hline $489^{a}$ & 3 & - & $25,42^{\mathrm{b}}, 45$ & - & - & Punjabi \\
\hline 743 & 3 & - & $40,44^{b}, 62$ & - & Bone (60), leukemia (60) & Pathan \\
\hline 658 & 3 & - & $26,44^{\mathrm{b}}, 50$ & - & - & Punjabi \\
\hline $377^{\mathrm{a}}$ & 3 & - & $31,50^{\mathrm{b}}, 85$ & - & $\begin{array}{l}\text { Thyroid (59), intestine (70), } \\
\text { bladder (75), liver }\end{array}$ & Punjabi \\
\hline $550^{d}$ & 3 & - & $50^{\mathrm{b}}, 55,>50$ & - & Lung, unknown & Punjabi \\
\hline 372 & $3(1)$ & - & $21 / 21^{b}, 29,45$ & - & $\begin{array}{l}\text { Squamous cell carcinoma } \\
\text { scalp }(22)^{b}\end{array}$ & Pathan \\
\hline $626^{\mathrm{a}}$ & $3(1)$ & - & $35^{\mathrm{b}}, 36 / 37,42$ & - & - & Balochi \\
\hline 389 & $3(1)$ & - & $22 / 32,42,48^{\mathrm{b}}$ & - & Brain (36), uterus (70) & Punjabi \\
\hline $247^{\mathrm{a}}$ & 4 & - & $27,28^{\mathrm{b}}, 40,42$ & - & Uterus $(31,55)$ & Siriaki \\
\hline $652^{\mathrm{a}}$ & 4 & - & $31^{b}, 33,42,50$ & - & - & Punjabi \\
\hline 362 & 4 & - & $31,32,35^{\mathrm{b}}, 45$ & - & Liver (>40), abdomen & Pathan \\
\hline $399^{\mathrm{a}}$ & 4 & - & $43,44^{\mathrm{b}}, 50, ?$ & - & $\begin{array}{l}\text { Abdomen (45), lung (45), } \\
\text { prostate (53) }\end{array}$ & Punjabi \\
\hline $338^{\mathrm{a}}$ & 4 & - & $30,40,44,48^{b}$ & - & Stomach (73) & Punjabi \\
\hline $408^{\mathrm{a}}$ & $4(1)$ & - & $24^{b}, 31 / 31,33,50$ & - & $\begin{array}{l}\text { Abdomen (54), } \\
\text { esophagus (74) }\end{array}$ & Punjabi \\
\hline 521 & $4(1)$ & - & $25 / 38^{b}, 27,33,70$ & - & $\begin{array}{l}\text { Stomach }(60,65) \text {, lung, } \\
\text { unknown }\end{array}$ & Punjabi \\
\hline 653 & 5 & - & $24,32,35^{\mathrm{b}}, 37,50$ & - & Colon (42), throat (66) & Punjabi \\
\hline 734 & 5 & - & $37,38^{\mathrm{b}}, 55, ?, ?$ & - & - & Punjabi \\
\hline 296 & $7(1)$ & - & $21^{\mathrm{b}},<30,34,43,44 / 44,51,52$ & - & - & Punjabi \\
\hline 439 & 8 & - & $30,<35,40^{\mathrm{b}}, 40,40,40,>50,>65$ & - & $\begin{array}{l}\text { Uterus (40), prostate, } \\
\text { unknown }\end{array}$ & Punjabi \\
\hline $249^{a}$ & $8(1)$ & - & $30 / 31^{\mathrm{b}}, 37,45, ?, ?, ?, ?, ?$ & - & - & Punjabi \\
\hline $619^{a}$ & 1 & 1 & $30^{b}$ & $>50$ & - & Punjabi \\
\hline 646 & 1 & 1(1) & $34^{\mathrm{b}}$ & $36^{b}$ & - & Punjabi \\
\hline 748 & 1 & 1(1) & $47^{b}$ & $52^{b}$ & Skin (45), liver (50) & Punjabi \\
\hline 542 & 1 & $2(1)$ & $40^{b}$ & $46^{\mathrm{b}}, 70$ & Leukemia (74) & Punjabi \\
\hline $210^{\mathrm{a}}$ & 1 & 4 & $45^{b}$ & $?, ?, ?, ?$ & $\begin{array}{l}\text { Brain (32), abdomen, lung, } \\
\text { leukemia }\end{array}$ & Punjabi \\
\hline $241^{\mathrm{a}}$ & 2 & 1 & $29^{\mathrm{b}}, 64$ & 35 & Lymphoma & Punjabi \\
\hline $598^{\mathrm{a}}$ & 2 & 1 & $30^{\mathrm{b}}, 56$ & $>50$ & $\begin{array}{l}\text { Stomach }(>50,>50) \\
\text { tongue }(>50)\end{array}$ & Punjabi \\
\hline 463 & 2 & 1 & $35^{\mathrm{b}}, 58$ & 48 & - & Punjabi \\
\hline 481 & 2 & 1 & $<25,47^{\mathrm{b}}$ & 36 & Lung (55), uterus & Punjabi \\
\hline 621 & 2 & 1 & $50,>60$ & $50^{b}$ & Prostate (65) & Punjabi \\
\hline $211^{\mathrm{a}}$ & 2 & $1(1)$ & $26^{\mathrm{b}}, 50$ & 50 & - & Punjabi \\
\hline $415^{\mathrm{a}}$ & 2 & 1(1) & $34,35^{\mathrm{b}}$ & $35^{b}$ & $\begin{array}{l}\text { Leukemia (45), } \\
\text { unknown }\end{array}$ & Punjabi \\
\hline 679 & 2 & $1(1)$ & $28^{\mathrm{b}},<33$ & $49^{b}$ & - & Punjabi \\
\hline$N 4^{\mathrm{a}}$ & 2 & $1(1)$ & $41^{b}, 45$ & 45 & Uterus (38) & Punjabi \\
\hline
\end{tabular}


Table 3 Characteristics of the 133 families with deleterious BRCA1/2 mutations (Continued)

\begin{tabular}{|c|c|c|c|c|c|c|}
\hline \multirow[t]{2}{*}{ Family } & \multicolumn{2}{|c|}{ No. of cancers } & \multicolumn{2}{|c|}{ Age at onset (years) } & \multirow{2}{*}{$\begin{array}{l}\text { Other cancer(s)c } \\
\text { (age at onset in years) }\end{array}$} & \multirow[t]{2}{*}{ Ethnicity } \\
\hline & Female BC (Bilateral) & $\mathrm{OC}(\mathrm{OC}+\mathrm{BC})$ & $\mathrm{BC}$ & OC & & \\
\hline $488^{a}$ & 2 & $2(1)$ & $40^{\mathrm{b}}, 55$ & $42^{\mathrm{b}}, 45$ & - & Punjabi \\
\hline 555 & 2 & $2(1)$ & $29^{b}, 36$ & $36^{b},>50$ & $\begin{array}{l}\text { Leukemia (10), vocal } \\
\text { cord (45) }\end{array}$ & Punjabi \\
\hline 317 & 2 & $3(1)$ & 41,46 & $47^{b}, 52,55$ & Fallopian tube $(47)^{\mathrm{b}}$ & Punjabi \\
\hline $318^{\mathrm{a}}$ & 2 & $4(2)$ & $40,46^{b}$ & $40,42^{\mathrm{b}}, 44,58$ & Bladder $(50,50)$ & Pathan \\
\hline 442 & $2(1)$ & 2 & $34,50 / 50^{\mathrm{b}}$ & 28,52 & Leukemia (15) & Punjabi \\
\hline $283^{\mathrm{a}}$ & $2(1)$ & 3 & $34 / 38^{b}, 56$ & $54,55,65$ & - & Pathan \\
\hline $254^{\mathrm{a}}$ & 3 & 1 & $27,32^{\mathrm{b}}, 43$ & 41 & Brain & Punjabi \\
\hline 711 & 3 & 1 & $40^{\mathrm{b}}, ?, ?$ & $?$ & Gall bladder & Sindhi \\
\hline $445^{\mathrm{a}}$ & 3 & $1(1)$ & $44^{b},>60,73$ & 74 & Gall bladder & Punjabi \\
\hline 363 & 3 & 2 & $32,35,70$ & $47^{b}, ?$ & Lung (65), oral (70), liver & Kashmiri \\
\hline $329^{a}$ & 3 & 3 & $34^{\mathrm{b}}, 39, ?$ & $39,<50, ?$ & - & Punjabi \\
\hline $609^{\mathrm{a}}$ & $4(1)$ & 1 & $29^{\mathrm{b}}, 31,48 / 55,65$ & 30 & - & Mohajir \\
\hline $328^{\mathrm{a}}$ & 4 & 1 & $29,30^{\mathrm{b}}, 31,39$ & 55 & - & Punjabi \\
\hline 501 & 4 & 1 & $34^{\mathrm{b}}, 35,>50, ?$ & $?$ & Brain (42) & Punjabi \\
\hline 522 & 4 & $2(1)$ & $30^{\mathrm{b}},<40,45,45$ & 35,60 & Uterus (41) & Punjabi \\
\hline $611^{\mathrm{a}}$ & 4 & $2(1)$ & $31^{b}, 36,37,42$ & 50,55 & Blood (30) & Punjabi \\
\hline $523^{\mathrm{a}}$ & $3(1)$ & 4 & $39 / 46,40,53^{b}$ & $30,45,51,60$ & - & Punjabi \\
\hline $275^{a}$ & $4(1)$ & $1(1)$ & $34 / 40^{\mathrm{b}}, 40,42,50$ & 40 & - & Punjabi \\
\hline 469 & 5 & $1(1)$ & $29^{\mathrm{b}}, 29,<35,>35,>55$ & $32^{\mathrm{b}}$ & - & Mohajir \\
\hline $340^{\mathrm{a}}$ & 6 & 1 & $34^{\mathrm{b}}, 42,<50,<50,<50,<50$ & 54 & - & Balochi \\
\hline $574^{\mathrm{a}}$ & 6 & 2 & $32,32,35,45^{\mathrm{b}}, 48,>50$ & 48,55 & - & Mohajir \\
\hline $512^{\mathrm{a}}$ & $6(1)$ & 1 & $<25,<30,<40,46 / 55^{\mathrm{b}},<50,>50$ & $<50$ & Uterus $(<50)$ & Kashmiri \\
\hline $248^{\mathrm{a}}$ & 7 & $2(2)$ & $23^{\mathrm{b}},<25,34,<40,<46,<60, ?$ & $23^{b},<60$ & - & Punjabi \\
\hline $220^{\mathrm{a}}$ & 8 & $1(1)$ & $25,27^{\mathrm{b}},<30,53,58,63,77$ & $<30$ & - & Punjabi \\
\hline \multicolumn{7}{|c|}{ Families carrying BRCA1-LGRs ${ }^{\mathrm{a}}$} \\
\hline 229 & 1 & - & $28^{\mathrm{b}}$ & - & - & Punjabi \\
\hline 379 & 2 & - & $29,31^{\mathrm{b}}$ & - & Liver (38) & Punjabi \\
\hline 261 & 2 & - & $33,34^{b}$ & - & - & Punjabi \\
\hline 406 & 2 & - & $39^{\mathrm{b}}, 40$ & - & Abdomen (65) & Punjabi \\
\hline 498 & 2 & - & $40,41^{\mathrm{b}}$ & - & - & Siriaki \\
\hline 549 & 2 & - & $38,72^{b}$ & - & Unknown & Punjabi \\
\hline 314 & 6 & - & $32^{\mathrm{b}}, 42,56,70, ?, ?$ & - & $\begin{array}{l}\text { Uterus (54), pharynx } \\
\text { (59), brain (63), } \\
\text { abdomen }\end{array}$ & Punjabi \\
\hline 291 & 3 & 1 & $39,42,48$ & $48^{b}$ & Stomach, brain & Punjabi \\
\hline 719 & $3(1)$ & 1 & $>40,42^{b}, ?$ & $?$ & - & Punjabi \\
\hline \multicolumn{7}{|c|}{ Families carrying $B R C A 2$-small-range mutations } \\
\hline 330 & 1 & - & $29^{b}$ & - & $\begin{array}{l}\text { Lung }(48,58,66) \text {, } \\
\text { tongue }(55)\end{array}$ & Punjabi \\
\hline 206 & 1 & - & $30^{\mathrm{b}}$ & - & - & Pathan \\
\hline $540^{d}$ & 1 & - & $67^{\mathrm{b}}$ & - & - & Mohajir \\
\hline $207^{d}$ & 1 & - & $76^{\mathrm{b}}$ & - & Intestine (60) & Punjabi \\
\hline $295^{a}$ & $1(1)$ & - & $23 / 23^{b}$ & - & $\begin{array}{l}\text { Leukemia (49), } \\
\text { esophagus (50) }\end{array}$ & Punjabi \\
\hline
\end{tabular}


Table 3 Characteristics of the 133 families with deleterious BRCA1/2 mutations (Continued)

\begin{tabular}{|c|c|c|c|c|c|c|}
\hline \multirow[t]{2}{*}{ Family } & \multicolumn{2}{|c|}{ No. of cancers } & \multicolumn{2}{|c|}{ Age at onset (years) } & \multirow{2}{*}{$\begin{array}{l}\text { Other cancer(s) } \\
\text { (age at onset in years) }^{c}\end{array}$} & \multirow[t]{2}{*}{ Ethnicity } \\
\hline & Female BC (Bilateral) & $\mathrm{OC}(\mathrm{OC}+\mathrm{BC})$ & $\mathrm{BC}$ & OC & & \\
\hline N26 & 2 & - & $26^{\mathrm{b}}, 35$ & - & - & Pathan \\
\hline 602 & 2 & - & $31^{b}, 43$ & - & - & Punjabi \\
\hline 492 & 2 & - & $38^{\mathrm{b}}, 39$ & - & - & Mohajir \\
\hline 505 & 2 & - & $43,46^{b}$ & - & - & Pathan \\
\hline 713 & 2 & - & $35,56^{\mathrm{b}}$ & - & - & Kashmiri \\
\hline 700 & $2(1)$ & - & $35 / 43^{b}, 46$ & - & Throat (72) & Punjabi \\
\hline 627 & $2(1)$ & - & $42^{b}, 51 / 51$ & - & - & Pathan \\
\hline 545 & 3 & - & $35,36^{\mathrm{b}}, 47$ & - & $\begin{array}{l}\text { Brain (50), uterus (50), } \\
\text { bone (54) }\end{array}$ & Punjabi \\
\hline 497 & 3 & - & $51^{b}, 55,50$ & - & Brain & Siriaki \\
\hline $548^{d}$ & 3 & - & $45,50,69^{b}$ & - & - & Pathan \\
\hline 702 & 4 & - & $26,30^{b}, \leq 33,70$ & - & Throat (72) & Punjabi \\
\hline 579 & 4 & - & $35,49,<50,51^{b}$ & - & Oral (35), gall bladder (42) & Kashmiri \\
\hline $407^{d}$ & 4 & - & 31,45 (male), $45,54^{\mathrm{b}}$ & - & $\begin{array}{l}\text { Esophagus }(39,59), \\
\text { leukemia }(64)\end{array}$ & Mohajir \\
\hline 538 & 5 & - & $34^{b}, 38,45,50,58$ & - & $\begin{array}{l}\text { Retinoblastoma (3), } \\
\text { pancreas (75), liver (83) }\end{array}$ & Pathan \\
\hline 684 & 5 & - & $40^{\mathrm{b}}, 45,48,50,<57$ & - & $\begin{array}{l}\text { Throat }(<48,<82) \text {, } \\
\text { stomach }(53) \text {, } \\
\text { intestine }(60)\end{array}$ & Pathan \\
\hline 447 & $3(1)$ & 2 & $31^{b},<50,55 / 65$ & $50,>50$ & $\begin{array}{l}\text { Abdomen }(>50) \text {, } \\
\text { colon }(62), \\
\text { brain }(65)\end{array}$ & Punjabi \\
\hline 525 & $4(1)$ & 1 & $32,33 / 35^{\mathrm{b}},<50,60$ & $<45$ & Bladder (>60) & Mohajir \\
\hline 222 & 7 & $2(1)$ & $35,42,43,50,54^{\mathrm{b}}, 60, ?$ & $47,53^{b}$ & $\begin{array}{l}\text { Lung (60), prostate (70), } \\
\text { lung }\end{array}$ & Kashmiri \\
\hline
\end{tabular}

$B C$ breast cancer, $O C$ ovarian cancer, Unknown cancer phenotype is not known

${ }^{a}$ Mutations previously described $[11,18] ;{ }^{b}$ Proband; ${ }^{c}$ Age at cancer diagnosis is mentioned along with cancer phenotype. For relatives with unknown age at cancer onset, only cancer phenotype is mentioned; ${ }^{\mathrm{d}}$ Families with male breast cancer

times more frequent than $B R C A 2$ mutations. A similar distribution was observed in two Asian studies from South India [26] and Saudi Arabia [27] and most studies among white populations $[3-5,28]$. This is likely due to the predominance of recurrent BRCA1 mutations in these populations. Contradictory results were reported in other Asian studies from China, Hong Kong, Korea, and Indonesia, where BRCA2 mutations were observed at an equal or a higher frequency than BRCA1 mutations [6, 12, 15-17].

Among the 133 mutations identified in our study, 18 $B R C A 1$ and three BRCA2 mutations were recurrent, accounting for $68.2 \%$ and $34.8 \%$ of all mutations in BRCA1 and $B R C A 2$, respectively. The proportion of recurrent $B R C A 1$ mutations to the total number of identified BRCA1 mutations is higher than our previous report [10], which is likely due to the larger size of the present study. Of the identified recurrent mutations, the majority was also reported as recurrent mutations in other populations $[1,4,25]$, while few were exclusively identified in a specific ethnic group of Pakistan. Fourteen BRCA1 mutations (c.3770_3771del, c.5503C > T, c.4485$1 \mathrm{G}>\mathrm{A}, \quad$ c.2405_2406del, c.1793T $>\mathrm{G}, \quad$ c. $4508 \mathrm{C}>\mathrm{A}$, c.2603C $>\mathrm{G}, \quad$ c.3339_3341del, c.3598C $>\mathrm{T}, \quad$ c.5035del, c.5074+1G>A, c.5361_5362del, exon 1-2 deletion, and exon 21-24 deletion) and one BRCA2 mutation (c.92G $>A$ ) were identified only in the Punjabi ethnic group. One BRCA2 mutation (c.5682C>A) was found only in the Pathan ethnic group. Five other recurrent mutations were identified in more than one ethnic group. Our findings imply that a panel of ethnic specific recurrent mutations may be useful for initial screening of high-risk patients from these ethnic groups. Founder effects were previously suggested for six of the 18 recurrent BRCA1 mutations (c.3770_3771del, c.4065_4068del, c.4485-1G >A, c.4508C >A, c.5503C > T, exon 1-2 deletion) [9-11], while haplotype analyses of the remaining recurrent mutations have not been performed so far. 


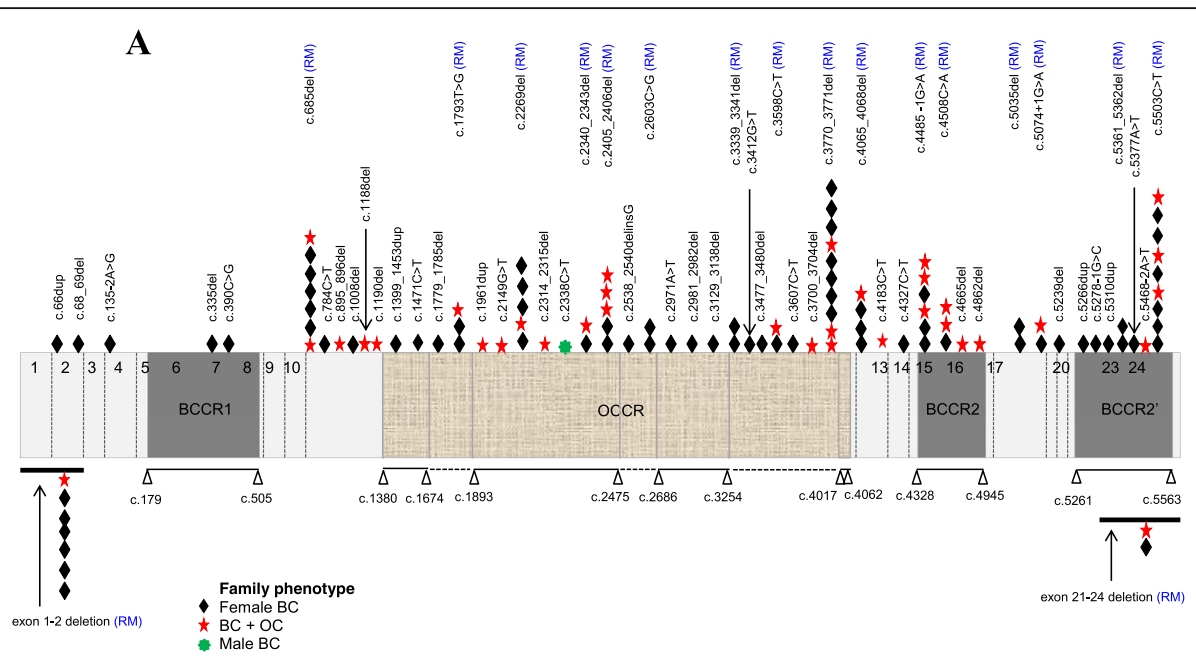

B

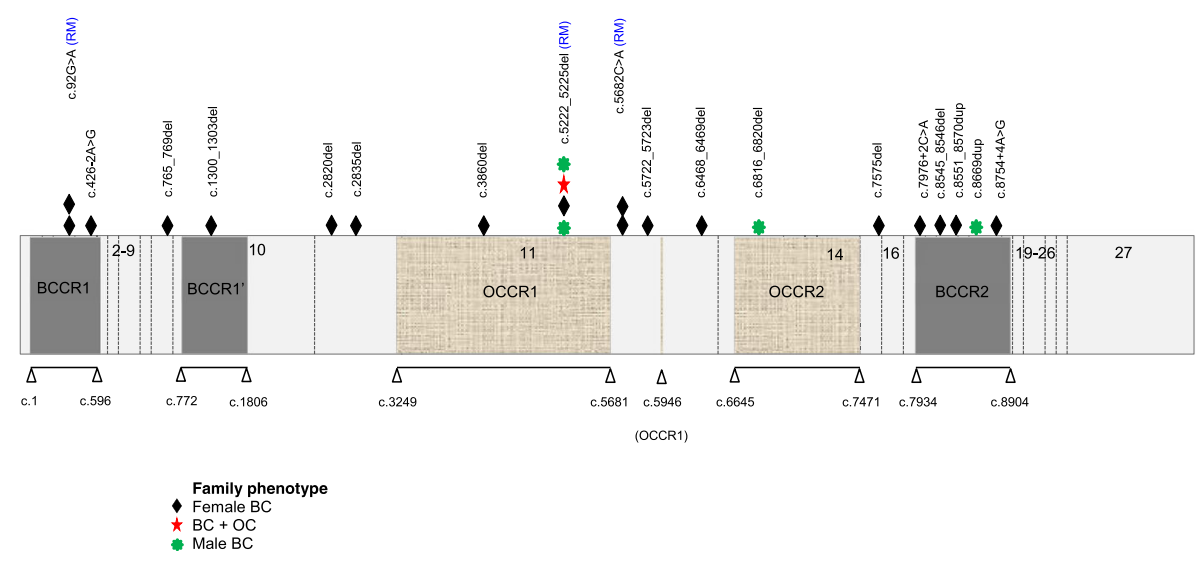

Fig. 2 Distribution of deleterious germline mutations identified in Pakistani breast/ovarian cancer families across the BRCA1 and BRCA2 genes. Recurrent mutations are marked with (RM). The distribution of breast cancer (BC) and ovarian cancer (OC) in families according to the position of the mutations in BRCA1 (a) and BRCA2 (b) is shown. Regions inferred to be breast cancer cluster regions (BCCRs) and ovarian cancer cluster regions (OCCRs) according to Rebbeck and colleagues [24] are shown at the bottom

The high percentage of recurrent $B R C A 1$ mutations facilitates the development of a local, economical, and efficient ethnic-specific genetic testing strategy in Pakistan.

$B R C A 1 / 2$ mutations were identified in $24.7 \%$ of Pakistani breast cancer families. This frequency is higher than that from our initial report (17\%) [10], probably due to the larger study size and comprehensive mutation analyses of both genes. This frequency is also higher than those from other Asian reports from Hong Kong, Malaysia, and Korea, ranging from $9.4 \%$ to $21.7 \%$ [6-8, $16,17]$. These findings further support the notion that the $B R C A 1 / 2$ mutation frequencies vary among different populations. Our data are similar to those reported in white populations, ranging from $17.6 \%$ to $29.8 \%[1,2,4]$. We found the highest mutation frequency in breast and ovarian cancer families (55.4\%), in agreement with previous studies from Pakistan [10], Korea [16], and studies in white populations $[4,28]$. We observed a 2.52 fold (53.8\% vs. $21.3 \%$ ) increased occurrence of BRCA1 mutations in breast and ovarian cancer families compared to breast cancer only families, in line with previous reports $[1,4,6,28]$. Our findings support the notion that the presence of ovarian cancer in Pakistani breast cancer families increases the likelihood for the occurrence of BRCA1 mutation.

In the present study on 27 families with male breast cancer, a $B R C A 1 / 2$ mutation frequency of approximately $19 \%$ was observed, with $B R C A 2$ mutations being about 4-times more common than BRCA1 mutations. Our data are in line with previous studies [4, 14]. This observed frequency is higher than that reported in our initial much smaller study, in which no mutations were identified [10]. In agreement with the National Comprehensive Cancer Network (NCCN) guidelines, our data also 
warrant $B R C A 1 / 2$ testing in families with male breast cancer (NCCN Guidelines Version 2.2019).

The main strength of this study is its large size of 539 high-risk families, the comprehensive screening of both genes for small-range mutations and LGRs using highly sensitive methods (allowing the identification of recurrent $B R C A 1 / 2$ mutations in the Pakistani population and the more accurate estimation of $B R C A 1 / 2$ mutation frequencies among high-risk families), and the confirmations of mutations in an independent patient's sample. However, our study also has some limitations. Participants were recruited at one tertiary care cancer center in Lahore, which may have introduced selection bias. Families belonging to Punjabi and Pathan ethnic groups are over-represented and, therefore, mutations in these groups may be over-represented. Nevertheless, Punjabi (44.7\%) and Pathan $(15.4 \%)$ are the most common ethnic groups reported in Pakistan (The World Factbook). Further, our data are based on self-reported ethnicity of study participants, which may lead to a misclassification of the ethnic origin of some of them.

\section{Conclusions}

In summary, our study showed that $B R C A 1 / 2$ mutations account for $24.7 \%$ of high-risk breast cancer patients in Pakistan. Our results have important clinical implications, such as personalized treatment with platinum-based or PARP-inhibitor therapy for breast/ovarian cancer patients carrying a pathogenic $B R C A 1 / 2$ mutation and early detection, surgical prevention, and chemoprevention strategies for their unaffected $B R C A 1 / 2$ mutation positive relatives. Overall, BRCA1/2 mutations account for one in four patients with a family history of breast cancer/breast and ovarian cancer, one in five patients with male breast cancer, and one in eight patients with early-onset breast cancer. Eighteen mutations in BRCA1 and three in BRCA2 were recurrent and accounted for $68.2 \%$ and $34.8 \%$ of all identified mutations in BRCA1 and BRCA2, respectively. Our data suggest that $B R C A 1$ testing may be justified for families with multiple female breast cancers, breast and ovarian cancer or early-onset breast cancer and BRCA2 testing for families with male breast cancer from Pakistan. Our findings will help in tailoring cost-effective genetic testing approach for the high-risk Pakistani population or for individuals of Pakistani origin residing in other countries.

\section{Abbreviations}

BIC: Breast cancer information core; HGVS: Human genome variation society; LGRs: Large genomic rearrangements; LOVD: The Leiden open variation database; SKMCH\&RC: Shaukat Khanum Memorial Cancer Hospital and Research Centre

\section{Acknowledgements}

We are grateful to all study subjects for their participation in this study. We thank the clinicians (Neelam Siddiqui, Mazhar Ali Shah, Narjis Muzaffar, Usman Ahmad, Umm E Kalsoom, Amir Ali Syed, Huma Majeed, Zulqarnain Chaudhry, Muhammad Asad Parvaiz, and Amina Khan) for their help in recruitment of study participants. We thank Jörg Hoheisel for critical reading of the manuscript.

\section{Authors' contributions}

MUR contributed to conception and design of the study, patient recruitment and data acquisition. In addition, he was involved in data analysis, interpretation and in drafting and revising the manuscript. NM performed the molecular analyses and contributed to data analysis and interpretation. He was also involved in writing the first draft of the manuscript. HN, FAK and SG performed the molecular analysis. MH and SF were involved in patient recruitment and clinical data acquisition. AA was involved in the recruitment of study subjects, clinical data collection and revising the manuscript. AL was involved in the pathological data acquisition and interpretation. UH contributed to conception and design of the study, data analysis and interpretation and led the writing of the manuscript. All authors read and approved the final manuscript.

\section{Funding}

The study was supported by the Shaukat Khanum Memorial Cancer Hospital and Research Centre (grant number ONC-BRCA-002) and the German Cancer Research Center.

\section{Availability of data and materials}

The datasets used and/or analyzed during the current study are available from the corresponding author on reasonable request.

\section{Ethics approval and consent to participate}

This study was conducted in accordance with the Declaration of Helsinki and Good Clinical Practice guidelines. It was approved by the ethics committee of Shaukat Khanum Memorial Cancer Hospital and Research Centre (SKMCH\&RC), Lahore Pakistan. The ethics committee name is the "Institutional Review Board". The approval number is ONC-BRCA-002. Written informed consent was obtained from all study participants.

\section{Consent for publication}

Not applicable

\section{Competing interests}

The authors declare that they have no competing of interests.

\section{Author details}

${ }^{1}$ Basic Sciences Research, Shaukat Khanum Memorial Cancer Hospital and Research Centre (SKMCH\&RC), Lahore, Pakistan. ${ }^{2}$ Molecular Genetics of Breast Cancer, German Cancer Research Center (DKFZ), Im Neuenheimer Feld 580, 69120 Heidelberg, Germany. ${ }^{3}$ Clinical Research Office, SKMCH\&RC, Lahore, Pakistan. ${ }^{4}$ Leibniz Institute on Aging - Fritz Lipmann Institute, Jena, Germany. ${ }^{5}$ Levine Cancer Institute, Charlotte, USA. ${ }^{6}$ Pathology Department, SKMCH\&RC, Lahore, Pakistan.

Received: 23 May 2019 Accepted: 2 September 2019

Published online: 11 September 2019

\section{References}

1. de Juan JI, Garcia Casado Z, Palanca Suela S, Esteban Cardenosa E, Lopez Guerrero JA, Segura Huerta A, et al. Novel and recurrent BRCA1/BRCA2 mutations in early onset and familial breast and ovarian cancer detected in the Program of Genetic Counseling in Cancer of Valencian Community (eastern Spain). Relationship of family phenotypes with mutation prevalence. Fam Cancer. 2013;12:767-77.

2. James PA, Sawyer S, Boyle S, Young MA, Kovalenko S, Doherty R, et al. Large genomic rearrangements in the familial breast and ovarian cancer gene BRCA1 are associated with an increased frequency of high risk features. Fam Cancer. 2015;14:287-95.

3. Janavicius R, Rudaitis V, Mickys U, Elsakov P, Griskevicius L. Comprehensive BRCA1 and BRCA2 mutational profile in Lithuania. Cancer Genet. 2014;207: 195-205.

4. Stegel V, Krajc M, Zgajnar J, Teugels E, De Greve J, Hocevar M, et al. The occurrence of germline BRCA1 and BRCA2 sequence alterations in Slovenian population. BMC Med Genet. 2011;12:9. 
5. Thomassen M, Gerdes AM, Cruger D, Jensen PK, Kruse TA. Low frequency of large genomic rearrangements of BRCA1 and BRCA2 in western Denmark. Cancer Genet Cytogenet. 2006;168:168-71.

6. Han SA, Kim SW, Kang E, Park SK, Ahn SH, Lee MH, et al. The prevalence of BRCA mutations among familial breast cancer patients in Korea: results of the Korean Hereditary Breast Cancer study. Fam Cancer. 2013;12:75-81.

7. Kang P, Mariapun S, Phuah SY, Lim LS, Liu J, Yoon SY, et al. Large BRCA1 and BRCA2 genomic rearrangements in Malaysian high risk breast-ovarian cancer families. Breast Cancer Res Treat. 2010;124:579-84.

8. Kwong A, Shin VY, Au CH, Law FB, Ho DN, Ip BK, et al. Detection of Germline Mutation in Hereditary Breast and/or Ovarian Cancers by NextGeneration Sequencing on a Four-Gene Panel. J Mol Diagn. 2016;18:580-94.

9. Liede A, Malik IA, Aziz Z, Rios Pd Pde L, Kwan E, Narod SA. Contribution of BRCA1 and BRCA2 mutations to breast and ovarian cancer in Pakistan. Am J Hum Genet. 2002;71:595-606.

10. Rashid MU, Zaidi A, Torres D, Sultan F, Benner A, Naqvi B, et al. Prevalence of BRCA1 and BRCA2 mutations in Pakistani breast and ovarian cancer patients. Int J Cancer. 2006;1 19:2832-9.

11. Rashid MU, Muhammad N, Amin A, Loya A, Hamann U. Contribution of BRCA1 large genomic rearrangements to early-onset and familial breast/ ovarian cancer in Pakistan. Breast Cancer Res Treat. 2017;161:191-201.

12. Purnomosari D, Pals G, Wahyono A, Aryandono T, Manuaba TW, Haryono SJ, et al. BRCA1 and BRCA2 germline mutation analysis in the Indonesian population. Breast Cancer Res Treat. 2007;106:297-304.

13. Sugano K, Nakamura S, Ando J, Takayama S, Kamata H, Sekiguchi I, et al. Cross-sectional analysis of germline BRCA1 and BRCA2 mutations in Japanese patients suspected to have hereditary breast/ovarian cancer. Cancer Sci. 2008;99:1967-76.

14. Son BH, Ahn SH, Kim SW, Kang E, Park SK, Lee MH, et al. Prevalence of BRCA1 and BRCA2 mutations in non-familial breast cancer patients with high risks in Korea: the Korean Hereditary Breast Cancer (KOHBRA) Study. Breast Cancer Res Treat. 2012;133:1143-52.

15. Zhang J, Sun J, Chen J, Yao L, Ouyang T, Li J, et al. Comprehensive analysis of BRCA1 and BRCA2 germline mutations in a large cohort of 5931 Chinese women with breast cancer. Breast Cancer Res Treat. 2016;158:455-62.

16. Kang E, Park SK, Lee JW, Kim Z, Noh WC, Jung Y, et al. KOHBRA BRCA risk calculator (KOHCal): a model for predicting BRCA1 and BRCA2 mutations in Korean breast cancer patients. J Hum Genet. 2016;61:365-71.

17. Kang E, Seong MW, Park SK, Lee JW, Lee J, Kim LS, et al. The prevalence and spectrum of BRCA1 and BRCA2 mutations in Korean population: recent update of the Korean Hereditary Breast Cancer (KOHBRA) study. Breast Cancer Res Treat. 2015;151:157-68.

18. Rashid MU, Muhammad N, Bajwa S, Faisal S, Tahseen M, Bermejo JL, et al. High prevalence and predominance of BRCA1 germline mutations in Pakistani triple-negative breast cancer patients. BMC Cancer. 2016;16:673.

19. Rashid MU, Muhammad N, Faisal S, Amin A, Hamann U. Deleterious RAD51C germline mutations rarely predispose to breast and ovarian cancer in Pakistan. Breast Cancer Res Treat. 2014;145:775-84.

20. Rashid MU, Muzaffar M, Khan FA, Kabisch M, Muhammad N, Faiz S, et al. Association between the Bsml Polymorphism in the Vitamin D Receptor Gene and Breast Cancer Risk: Results from a Pakistani Case-Control Study. PLoS One. 2015;10:e0141562.

21. Arnold N, Gross E, Schwarz-Boeger U, Pfisterer J, Jonat W, Kiechle M. A highly sensitive, fast, and economical technique for mutation analysis in hereditary breast and ovarian cancers. Hum Mutat. 1999;14:333-9.

22. Gross E, Arnold N, Pfeifer K, Bandick K, Kiechle M. Identification of specific BRCA1 and BRCA2 variants by DHPLC. Hum Mutat. 2000;16:345-53.

23. Claes K, Poppe B, Machackova E, Coene I, Foretova L, De Paepe A, et al. Differentiating pathogenic mutations from polymorphic alterations in the splice sites of BRCA1 and BRCA2. Genes Chromosomes Cancer. 2003;37: 314-20

24. Rebbeck TR, Mitra N, Wan F, Sinilnikova OM, Healey S, McGuffog L, et al. Association of type and location of BRCA1 and BRCA2 mutations with risk of breast and ovarian cancer. JAMA. 2015;313:1347-61.

25. Rebbeck TR, Friebel TM, Friedman E, Hamann U, Huo D, Kwong A, et al. Mutational spectrum in a worldwide study of 29,700 families with BRCA1 or BRCA2 mutations. Hum Mutat. 2018;39:593-620.

26. Vaidyanathan K, Lakhotia S, Ravishankar HM, Tabassum U, Mukherjee G, Somasundaram K. BRCA1 and BRCA2 germline mutation analysis among Indian women from south India: identification of four novel mutations and high-frequency occurrence of 185delAG mutation. J Biosci. 2009;34:415-22.
27. Bu R, Siraj AK, Al-Obaisi KA, Beg S, Al Hazmi M, Ajarim D, et al. Identification of novel BRCA founder mutations in Middle Eastern breast cancer patients using capture and Sanger sequencing analysis. Int J Cancer. 2016;139:1091-7.

28. Machackova E, Foretova L, Lukesova M, Vasickova P, Navratilova M, Coene I, et al. Spectrum and characterisation of BRCA1 and BRCA2 deleterious mutations in high-risk Czech patients with breast and/or ovarian cancer. BMC Cancer. 2008:8:140

\section{Publisher's Note}

Springer Nature remains neutral with regard to jurisdictional claims in published maps and institutional affiliations.

\section{Ready to submit your research? Choose BMC and benefit from:}

- fast, convenient online submission

- thorough peer review by experienced researchers in your field

- rapid publication on acceptance

- support for research data, including large and complex data types

- gold Open Access which fosters wider collaboration and increased citations

- maximum visibility for your research: over $100 \mathrm{M}$ website views per year

At BMC, research is always in progress.

Learn more biomedcentral.com/submissions 